\title{
Violencia contra la mujer y regulación jurídica del femicidio en Ecuador*
}

\section{Violence Against Women And Legal Regulation On Femicide In Ecuador}

\author{
Lianet Goyas Cespedes** \\ Silvia Patricia Zambrano Noles*** \\ IRIS CABANES ESPINO****
}

\section{RESUMEN}

La violencia contra las mujeres es reconocida como un problema social a nivel mundial, aunque es dificil de expresar en cifras por la cantidad de casos que no son denunciados; según datos de la Encuesta Nacional sobre Relaciones Familiares y Violencia de Género, en Ecuador la violencia por razón de género ha afectado a seis de cada diez mujeres. Ante esta situación, la sociedad civil y los gobiernos trazan estrategias para la erradicación de todas las formas de violencia contra la mujer, entre las que se encuentra la tipificación del femicidio en las legislaciones de varios paises latinoamericanos, pero esto no ha parado las muertes de mujeres por razón de su género. Todo esto motiva el análisis de la regulación en varios paises de América Latina respecto a la figura del femicidio, con especial referencia en Ecuador, que se presenta en este artículo como resultado del proyecto de investigación "Deberes y derechos ciudadanos", que se desarrolla en la Universidad Metropolitana del Ecuador.

Palabras clave: discriminación por el género, violencia contra la mujer, femicidio, feminicidio.

\begin{abstract}
Violence against women is a worldwide social problem, although gender violence against women is difficult to express in terms of numbers or cases since they are not reported, according to data from the national survey on family relations and gender violence, gender violence in Ecuador affects 6 out of 10 women. In the face of this situation, civil society and government outline strategies for the eradication of all forms of violence against women, including the definition of femicide in the legislations of several Latin American countries, but this alone has had no impact on the death toll of women by gender reasons. Therefore there is a need for an analysis on the regulations in several countries of Latin America on the numbers of femicides with special reference to Ecuador, which is presented in this article as a result of Duties and Citizen Rights Project that was developed in the Metropolitan University of Ecuador
\end{abstract}

Keywords: gender discrimination, violence against women, femicide.

*Artículo de investigación recibido el 18 de junio de 2017 y aceptado para publicación el 18 de enero de 2018

** Profesora Investigadora en la Universidad Metropolitana del Ecuador, (Sede Machala). (lianetgoyascespedes@ gmail.com) orcid.org/0000-0002-6634-2308

*** Profesora Investigadora en la Universidad Metropolitana del Ecuador, (Sede Machala). (silzam_79@hotmail.com) orcid.org/0000-0003-2696-6447

**** Profesora Investigadora en la Universidad Metropolitana del Ecuador, (Sede Guayaquil). (iriscabanes2016@ gmail.com) orcid.org/0000-0002-9990-0142 
SUMARIO: 1. Introducción / 2. La discriminación por el género como génesis y manifestación de todos los tipos de violencia contra la mujer / 3. Las cifras de violencia contra la mujer resultan alarmantes / 4. Creación de un Ministerio de Igualdad de Oportunidades entre mujeres y varones / 5. Los esfuerzos institucionales por eliminar la violencia contra la mujer en Ecuador / 6. Tipificación del femicidio o feminicidio en la legislación de países de América Latina / 7. La tipificación del femicidio en Ecuador / 8. Conclusiones / 9. Referencias

\section{INTRODUCCIÓN}

La situación de desventaja en que ha estado la mujer ha sido motivada por una estructura de poder que ha imperado en las culturas patriarcales, asignándole roles que perpetúan la dominación de los hombres y su subordinación, y que garantizan que se vuelvan invisibles los aportes de las mujeres a la sociedad. Los estereotipos creados a partir de supuestos papeles determinados por la biología de los sexos han justificado la discriminación a todo nivel, la desigualdad en el acceso a oportunidades y a los servicios de educación, salud y empleo, así como la exclusión de la que son sujetos. Como señala Magdalena Herdoíza: "Esta es una deuda del Estado con las mujeres, principalmente para con las mujeres indigenas, rurales y afrodescendientes". ${ }^{1}$

Las diferencias que persisten en las relaciones sociales entre hombres y mujeres en la sociedad generan manifestaciones de misoginia $\mathrm{y}$, como su consecuencia más deplorable, femicidios que cobra anualmente miles de vidas de mujeres. Como expresa Ana Carcedo: "La muerte de mujeres a manos de sus esposos, amantes, padres, novios, pretendientes, conocidos o desconocidos no es el producto de casos inexplicables o de conducta desviada o patológica. Por el contrario, es el producto de un sistema estructural de opresión. Estas muertes son femicidios, la forma más extrema de terrorismo sexista, motivado, mayoritariamente, por un sentido de posesión y control sobre las mujeres". ${ }^{2}$

\footnotetext{
${ }^{1}$ Herdoiza, Magdalena, Construyendo Igualdad en la educación superior. Fundamentación y lineamientos para transversalizar los ejes de igualdad y ambiente, Senescyt/Unesco, Quito, 2015, p. 46.

${ }^{2}$ Carcedo, Ana, Femicidio en Costa Rica 1990-1999, Inamu, Colección Teórica Número 1, San José, Costa Rica, 2000, p. 13.
} 
En ese sentido, son muchos los países que han tipificado esa figura en sus legislaciones, sin embargo, es un fenómeno que sigue en aumento; muestra de ello es que en los primeros días del año 2017 en Ecuador, no obstante tener regulado este tipo penal, ha crecido tanto la cifra de femicidios que el Consejo Nacional para la Igualdad de Género publicó un Pronunciamiento ${ }^{3}$ en el que se hace un llamado a las instituciones estatales para redoblar esfuerzos que eviten esos actos en el país.

Teniendo en cuenta todo lo anterior, en este trabajo se pretende valorar la posición adoptada por Ecuador en cuanto al enfrentamiento con el femicidio, a partir del análisis de las acciones institucionales realizadas para su prevención y erradicación, así como la comparación con su regulación en otros ordenamientos jurídicos latinoamericanos, para así poder fundamentar las limitaciones del marco legal vigente en el Código Orgánico Integral Penal.

\section{LA DISCRIMINACIÓN POR EL GÉNERO COMO GÉNESIS Y MANIFESTACIÓN DE} TODOS LOS TIPOS DE VIOLENCIA CONTRA LA MUJER

La discriminación de la mujer ha sido institucionalizada a través de los siglos a nivel mundial. Al colocarla en una situación desventajosa en todos los ámbitos de las relaciones sociales, también se le hace blanco de las diversas formas de violencia. Depender económicamente de los hombres, no tener un adecuado nivel educacional y cultural, no poder acceder a determinados empleos, tener asignada la crianza de los hijos y el cuidado de la familia, entre otras cuestiones, han hecho que las mujeres sean violentadas en sus relaciones públicas y privadas.

En Ecuador, según el censo del 2010, 50.4\% de la población corresponde a mujeres. Respecto a la educación, en diciembre de 2013 la prevalencia del analfabetismo a nivel nacional era de 6.71\%, mientras que para las mujeres el promedio se elevaba un punto porcentual $7.78 \% .^{4}$ (Ministerio Coordinador de Desarrollo Social, 2014).

En cuanto a la participación en la vida económica, los datos de diciembre de 2013 muestran que el subempleo y desempleo impactan principalmente a las mujeres, pues afectan a $58.70 \%$ en la población femenina mayor de 10 años, en comparación con el $44.8 \%$ en los hombres.

\footnotetext{
${ }^{3}$ Consejo Nacional para la Igualdad de Género. Disponible en: http://igualdadgenero.gob.ec/images/docs/Comunicac-2017/Pronunciamiento\%20Enero\%202017.jpg. Consultado el 1 de febrero de 2017.

${ }^{4}$ Comisión de Transición hacia el Consejo de Mujeres y la Igualdad de Género, 2014.
} 
Por su parte, la encuesta específica sobre uso del tiempo, realizada en el 2012 en el marco de colaboración Instituto Nacional Estadísticas y Censo (INEC) y la Comisión de Transición hacia el Consejo de Mujeres y la Igualdad de Género (CDT), muestra que a nivel nacional el tiempo total de trabajo es de 66:27 horas por semana. Las mujeres reflejan una mayor carga en el trabajo no remunerado, con una diferencia de 22:40 horas frente a los hombres, señalando que esta diferencia se debe a la asignación cultural de las responsabilidades domésticas y de cuidado al género femenino. ${ }^{5}$

\section{LAS CIFRAS DE VIOLENCIA CONTRA LA MUJER RESULTAN ALARMANTES}

En la Convención Interamericana para Prevenir, Sancionar y Erradicar la Violencia contra la Mujer, Convención de Belém do Pará, se define lo siguiente:

\section{Artículo 1}

Para los efectos de esta Convención debe entenderse por violencia contra la mujer cualquier acción o conducta, basada en su género, que cause muerte, daño o sufrimiento físico, sexual o psicológico a la mujer, tanto en el ámbito público como en el privado ${ }^{6}$.

En ese sentido, en el texto de la Convención Interamericana para Prevenir, Sancionar y Erradicar la Violencia contra la Mujer, Convención de Belém do Pará, se muestra preocupación porque "la violencia en que viven muchas mujeres de América, sin distinción de raza, clase, religión, edad o cualquier otra condición, es una situación generalizada". ${ }^{7}$

En Argentina, cada 30 horas una mujer es asesinada y cada año aumenta el número de femicidios. Entre 2008 y 2014 murieron 1808 mujeres asesinadas por la violencia machista. ${ }^{8}$ Las organizadoras de Ni Una Menos enviaron a los políticos cinco puntos a través de los cuales les exigen un compromiso público de aplicación de políticas específicas para combatir el femicidio:

\footnotetext{
${ }^{5}$ INEC. Disponible en: http://www.ecuadorencifras.gob.ec//documentos/web-inec/Uso_Tiempo/Presentacion_\%20 Principales_Resultados.pdf. Consultado el 20 de diciembre de 2016.

${ }^{6}$ Convención Interamericana para Prevenir, Sancionar y Erradicar la Violencia contra la Mujer, Convención de Belém do Pará. Disponible en: https://www.unicef.org/argentina/spanish/ar_insumos_ConvencionBelem.pdf. Consultado el 27 de enero de 2017.

7 Ibidem.

${ }^{8}$ Dato disponible en: http://www.feminicidio.net/articulo/ni-menos-la-sociedad-argentina-indignada-la-ola-femicidios-azota-al-pa\%C3\%ADs. Consultado el 20 de diciembre de 2016.
} 
1. Elaboración de estadísticas oficiales sobre violencia contra las mujeres y un índice de femicidios.

2. Creación de fueros regionales especializados en violencia contra las mujeres con competencia en materia civil y penal.

3. Reglamentación en su totalidad de la ley 26.485 y asignación de recursos.

4. Incorporación de la temática violencia contra las mujeres en todas las currículas educativas.

4. Creación de un Ministerio de lgualdad de Oportunidades entre MUJERES Y VARONES.

En México, según la oNU, siete mujeres son asesinadas cada día. Esta terrorífica cifra convierte al país azteca en uno de los integrantes del G-20 en el que las mujeres se encuentran más desprotegidas después de India, Arabia Saudita, Indonesia y Sudáfrica, según un análisis de TrustLaw para la Fundación Thomson Reuters. ${ }^{9}$

Además de la violencia generalizada, las mexicanas se enfrentan a la impunidad en sus asesinatos, al tráfico de personas, al trabajo en condiciones de esclavitud y a la falta de acceso a la educación, a la política y a la tierra. Entre 2013 y 2015, 6488 mujeres fueron asesinadas, según datos desprendidos de las estadísticas del INEGI. ${ }^{10}$

En el estudio realizado en Costa Rica por Ana Carcedo, que comprende de 1990 a 1999 se señala que, tomando en cuenta únicamente los homicidios de mujeres en los que se conocen las circunstancias en las que ocurrieron (autor, relación, forma de muerte), es decir, si se excluyen los indeterminados, el porcentaje de femicidios asciende a 70\% del total de los homicidios de mujeres. ${ }^{11}$

Cada año, entre 2004 y 2014, un promedio de 32 mujeres murió a manos de sus compañeros sentimentales en Costa Rica. En la gran mayoría de los casos - un 75 por ciento-, las víctimas nunca reportaron maltrato ante las autoridades ni presentaron denuncias formales de agresión contra sus asesinos. De las 316 muertes registradas esa década, sólo 16 víctimas tenían medidas de protección y únicamente 79 formularon denuncias por maltrato. ${ }^{12}$

\footnotetext{
9 Dato disponible en: http://www.economiahoy.mx/nacional-eAm-mx/noticias/7406635/03/16/Siete-mujeresmueren-al-dia-en-Mexico-victimas-de-la-violencia.html. Consultado el 2 de febrero de 2017.

$10 \mathrm{lbid}$

${ }^{11}$ Carcedo, Ana, op. cit., p. 34.

${ }^{12}$ Dato disponible en: http://www.ameliarueda.com/especiales/feminicidios/. Consultado el 2 de febrero de 2017.
} 
En Ecuador, seis de cada diez mujeres han sufrido algún tipo de violencia de género, es decir, $3260340 .{ }^{13}$ La familia debe constituir el espacio donde el ser humano se siente más seguro y en el que puede desarrollar su afectividad; sin embargo, es una realidad que en la actualidad las mujeres y otros miembros del núcleo familiar son abusados psicológica y físicamente en el interior de sus hogares. Según la Encuesta Nacional de Violencia contra las Mujeres, realizada por INEC en coordinación con la CDT en 2011, 76\% de estos actos de violencia es cometido por parejas o exparejas, quienes, en la mayoría de los casos, gozan de una legitimación arbitraria de este tipo de conductas y, por tanto, no son denunciadas. ${ }^{14}$ En lo que va del 2017 han matado a 15 mujeres; posiblemente sus parejas o exparejas las asesinaron. En el mismo periodo del 2016, se registraron ocho casos. ${ }^{15}$

En cuanto a los impactos del femicidio como expresión más brutal de la violencia contra la mujer, ha señalado Ana Carcedo:

Cuando una mujer muere a causa de la violencia de género es de esperar un fuerte impacto sobre las familias. Dado que la mayoría de las que fueron asesinadas en la década se encontraban en edad reproductiva, dejaron hijas e hijos menores. Por otra parte, cuando el homicida es el padre de estos niños y niñas, estos quedan sin su núcleo familiar. Además de la pérdida irreparable y el dolor que viven las y los hijos menores, las personas adultas de la familia interesadas en que se haga justicia tienen que enfrentar procesos judiciales para los que no están preparadas ni anímica ni legalmente, y para los que en ocasiones no cuentan con recursos de tiempo o económicos. Tienen que enfrentar también la frustración cuando el femicida evade la justicia o los Tribunales no actúan con la rigurosidad que la gravedad de estas muertes demanda. ${ }^{16}$

\footnotetext{
${ }^{13}$ Encuesta Nacional de Relaciones Familiares y Violencia de Género. Disponible en: http://www.igualdadgenero. gob.ec/estad\%C3\%ADsticas/57-encuesta-nacional-de-relaciones familiares-y-violencia-de-g\%C3\%A9nero.html. Consultado el 26 de diciembre de 2016. Este dato ha sido retomado en la exposición de motivos de la recientemente aprobada Ley Orgánica Integral para Prevenir y Erradicar la Violencia contra las Mujeres, de la Asamblea Nacional de la República del Ecuador. Ley Orgánica Integral para Prevenir y Erradicar la Violencia contra las Mujeres, Registro Oficial 175, Quito, 5 de febrero de 2018.

${ }^{14}$ Encuesta Nacional de Relaciones Familiares y Violencia de Género. Disponible en: http://www.igualdadgenero. gob.ec/estad\%C3\%ADsticas/57-encuesta-nacional-de-relaciones familiares-y-violencia-de-g\%C3\%A9nero.html. Consultado el 26 de diciembre de 2016.

15 El Universo. Disponible en: http://www.eluniverso.com/noticias/2017/01/24/nota/6014020/casos-femicidosecuador-se-han-duplicado-2017-segun-dinased. Consultado el 1 de febrero de 2017.

${ }^{16}$ Carcedo, Ana, op. cit., p. 48.
} 
5. LOS ESFUERZOS INSTITUCIONALES POR ELIMINAR LA VIOLENCIA CONTRA LA MUJER EN ECUADOR

Ecuador es signatario de los principales convenios y tratados del Sistema de Naciones Unidas, relacionados con la situación de la mujer, entre ellos la Convención sobre los Derechos Políticos de las Mujeres (1952), el Pacto Internacional de Derechos Civiles y Políticos (1966), la Convención para la Eliminación de Todas las Formas de Discriminación contra las Mujeres (CEDAW, 1979), la Plataforma de Acción de Beijing (1995) y los Objetivos de Desarrollo del Milenio contenidos en la Declaración del Milenio (ODM).

La Constitución de la República del Ecuador, de 2008, regula la protección integral de la mujer a través de varios artículos. Entre los más significativos se encuentran los referidos al reconocimiento de las mujeres embarazadas como personas y grupos de atención prioritaria, lo que se realiza en los artículos 35 y 43. Dentro de la protección que se brinda a estas personas y grupos se da un tratamiento especial a las mujeres al protegerlas cuando además han sido desplazadas arbitrariamente o están privadas de la libertad, lo que constituye una forma de prevenir la discriminación aditiva.

De igual forma, al reconocer y garantizar los derechos colectivos de comunas, comunidades, pueblos y nacionalidades indígenas, el artículo 57 establece que:

Art. 57: El Estado garantizará la aplicación de estos derechos colectivos sin discriminación alguna, en condiciones de igualdad y equidad entre mujeres y hombres.

En sus artículos 65, 108, 116, 176, 183, 210, 224 y 434, entre otros, se establece que el Estado promoverá la representación paritaria de hombres y mujeres en los cargos de nominación o designación de la función pública, y en los partidos y movimientos políticos. Asimismo, en materia de empleo los artículos 331, 332 y 333 establecen la obligación del Estado para garantizar a las mujeres la igualdad en el acceso al empleo.

Específicamente en cuanto a la protección contra la violencia, en el artículo 66, numeral 3, inciso b), se regula.

b) Una vida libre de violencia en el ámbito público y privado. El Estado adoptará las medidas necesarias para prevenir, eliminar y sancionar 
toda forma de violencia, en especial la ejercida contra las mujeres, niñas, niños y adolescentes, personas adultas mayores, personas con discapacidad y contra toda persona en situación de desventaja o vulnerabilidad; idénticas medidas se tomarán contra la violencia, la esclavitud y la explotación sexual.

Por último, en cuanto al análisis de la regulación constitucional, sin pretender agotarlo, debe señalarse que el artículo 70 establece:

Art. 70.- El Estado formulará y ejecutará políticas para alcanzar la igualdad entre mujeres y hombres, a través del mecanismo especializado de acuerdo con la ley, e incorporará el enfoque de género en planes y programas, y brindará asistencia técnica para su obligatoria aplicación en el sector público.

En cumplimiento con el mandato constitucional, en abril de 2014 se publicó la Agenda Nacional de las Mujeres y la Igualdad de Género 2014-2017, ${ }^{17}$ que es resultado de la concertación entre el Estado y la sociedad civil en torno a los derechos de género. Como señala Magdalena Herdoíza:

Esta agenda reivindica a los sujetos de derechos y sustenta, a partir de argumentos jurídicos y políticos, las intervenciones públicas orientadas a superar las brechas de desigualdad. Su propósito es la transformación de las relaciones sociales discriminatorias encaminadas a la construcción efectiva de un Estado en el que la igualdad sustantiva sea garantizada como una macro- política pública ${ }^{18}$.

El eje 2 de la Agenda se refiere a una vida libre de violencia, aglutina 15 lineamientos que se orientan a incrementar la cobertura del sistema de protección de víctimas de violencia, especializar al talento humano para una adecuada atención a las víctimas de violencia, fortalecer la ampliación y cobertura de los juzgados especializados, generar un subsistema integral de información, tipificar el femicidio como delito, la atención y sanción de los medios de comunicación y las TIC, sobre la difusión de contenidos y mensajes

\footnotetext{
${ }^{17}$ Consejo Nacional de Igualdad de Género, Agenda Nacional de las Mujeres y la lgualdad de Género 2014-2017, El Telégrafo, Quito, 2014.

${ }^{18}$ Herdoiza, Magdalena, op. cit., p. 51.
} 
machistas, sexistas, misóginos o que promuevan la violencia de género, entre otras cuestiones.

En el proceso de elaboración de la Agenda de Igualdad de Género por parte de la Comisión de Transición, en 2011, se produjo el Plan para la Igualdad, No Discriminación y Buen Vivir para las Mujeres Ecuatorianas: Marco Conceptual, Ruta Metodológica y Estrategia de Transversalización 2010-2014. ${ }^{19}$ El Plan establece como línea estratégica 6, "Erradicación de la violencia de género”, el acoso sexual, laboral y político, y el femicidio en los diversos ámbitos en la vida social, escolar, académica y doméstica.

En la rendición de cuentas del Ministerio Coordinador de Desarrollo Social, correspondiente al año 2014, se manifiesta que:

Para promover y garantizar una cultura de paz en la sociedad, el Gobierno Nacional consolidó un modelo de atención de calidad a las víctimas de violencia de género, a partir de estándares de atención e institucionalización de mecanismos de coordinación de los servicios. El proyecto desde el MCDS contribuyó en la construcción de la política pública de equidad género que se materializó en el Plan Nacional del Buen Vivir. En diciembre de 2014, se publicó la Norma Técnica de Atención a Víctimas de Violencia de Género para evitar la re victimización de las personas en los servicios de salud y del Sistema de Protección. Este fue un esfuerzo conjunto entre el Ministerio de Salud Pública, Consejo de la Judicatura y Fiscalía General del Estado. Se elaboraron guías de capacitación dirigidas a operadores de salud del MSP para la atención y direccionamiento de víctimas de violencia de género en el sistema de salud y se distribuyó material informativo sobre violencia de género en todas las salas de espera de los centros de salud. Además, se elaboraron guías para la atención en caso de delitos sexuales en ámbito educativo y se capacitó a 1.400 profesionales de los Departamentos de consejería estudiantil (dece) sobre protocolos y rutas de atención a víctimas de violencia en el sistema educativo. ${ }^{20}$

\footnotetext{
${ }^{19}$ Arboleda, Maria, Cuaderno de Trabajo Plan de Igualdad, No Discriminación y Buen Vivir para las Mujeres Ecuatorianas. Marco conceptual, ruta metodológica y estrategia de transversalización 2010-2014, primera edición, Comisión de Transición hacia el Consejo de las Mujeres y la Igualdad de Género, Quito, 2011.

${ }_{20}$ Ministerio Coordinador de Desarrollo Social. Disponible en: http://www.desarrollosocial.gob.ec/wp-content/ uploads/downloads/2015/03/RENDICION-CUENTAS-20141.pdf, pp. 15-16. Consultado el 26 de enero de 2016.
} 
En el informe de rendición de cuentas de la Fiscalía General del Estado, del año 2015, se expresa: "En este campo se crearon 70 unidades de investigación especializada de los delitos de violencia contra la mujer. Femicidio: Desde agosto del 2014, hemos obtenido 44 sentencias condenatorias con penas de hasta 40 años". ${ }^{21}$

Un hito trascendental en el enfrentamiento a todas las formas de violencia contra la mujer lo constituye la recientemente aprobada Ley Orgánica Integral para Prevenir y Erradicar la Violencia contra las Mujeres, cuya exposición de motivos expresa:

Esta Ley prevé de manera particular, enfocar la acción del Estado en la sensibilización y prevención de la violencia y con la participación de la ciudadanía, bajo el principio de corresponsabilidad. Estos dos actores deben garantizar a través de políticas, planes y programas, la transformación, de los patrones socioculturales y la erradicación de prácticas que naturalizan la violencia contra las mujeres. Esta Ley establece además tres componentes para la erradicación de la violencia: atención, protección y reparación de las mujeres víctimas de violencia para garantizar su seguridad e integridad y para retomar su proyecto de vida". ${ }^{22}$

\section{TiPifiCACIÓN DEL FEMICIDIO O FEMINICIDIO EN LA LEgISLACIÓN DE países de AmÉRICa latina}

Como ha planteado Ana Carcedo:

estas muertes causadas por la violencia intrafamiliar y la violencia sexual se originan en las relaciones desiguales de poder existentes en la sociedad entre la población masculina y la femenina, y se expresan al interior de las familias, en las relaciones de pareja y noviazgo y, en general, en las relaciones entre los géneros como relaciones de control de los hombres sobre las mujeres. Se trata de homicidios que no ocurren por casualidad, ni en los que las víctimas y los victimarios podrían ser

\footnotetext{
${ }^{21}$ Fiscalia General del Estado. Disponible en: fiscalia.gob.ec/informativo/Discurso_FGE_Rendicion_Cuentas_2016. pdf. Consultado el 26 de enero de 2017.

${ }^{22}$ Asamblea Nacional de la República del Ecuador, Ley Orgánica Integral para Prevenir y Erradicar la Violencia contra las Mujeres, Registro Oficial 175, Quito, 5 de febrero de 2018.
} 
indistintamente una mujer o un hombre. Se trata de homicidios en los que el hecho de que la víctima sea una mujer es una condición necesaria para que ocurran. ${ }^{23}$

En opinión de Roxana Arroyo:

La violencia contra la mujer no es un fenómeno aislado sino producto de una violencia estructural que impregna todo el tejido social estemos en tiempo de paz o de guerra, invisibilizar esto es negar las graves consecuencias que la violencia y la discriminación tienen en la vida de las mujeres y la responsabilidad de la Comunidad Internacional y los Estados en su ámbito interno para la prevención, sanción y erradicación de la violencia y la discriminación. ${ }^{24}$

\section{Como expresa Ana Isabel Garita:}

A pesar de que el concepto de femicidio no se gestó en América Latina, es en esta región en donde, en las últimas dos décadas, se ha producido un amplio debate sobre el concepto como efecto natural de la situación de vulnerabilidad y violencia en que se encuentran las mujeres $\mathrm{y}$, muy especialmente, por la ineficacia del sistema de justicia para contener y reprimir la muerte de mujeres. ${ }^{25}$

Tal como se expresa en el Informe sobre la Situación Mundial de la Prevención de la Violencia 2014: "Las leyes contra la violencia envían un mensaje claro a la sociedad acerca de los comportamientos inadmisibles y legitiman las acciones necesarias para garantizar la seguridad de la población en todo momento". ${ }^{26}$

\footnotetext{
${ }^{23}$ Carcedo, Ana, op. cit., p. 36.

${ }^{24}$ Arroyo, Roxana, Violencia Estructural de Género una Categoría Necesaria de Análisis para los Derechos Humanos de las Mujeres. Disponible en: http://observatoriojyg.org/index.php/280-doctrina/1-derecho-de-humanas/2igualdad/686-violencia-estructural-de-genero-una-categoria-necesaria-de-analisis-para-los-derechos-humanos-de-las-mujeres. Consultado el 26 de enero de 2017.

${ }^{25}$ Garita Vílchez, Ana Isabel, La regulación del delito de Femicidio/Feminicidio en América Latina y El Caribe, Secretariado de la Campaña del Secretario General de las Naciones Unidas ÚNETE para poner fin a la violencia contra las mujeres, Ciudad de Panamá, p. 15.

${ }^{26}$ ONU Informe sobre la Situación Mundial de la Prevención de la Violencia 2014, Washington, D.C., 2016, p. 40. Disponible en: https://oig.cepal.org/sites/default/files/informe_sobre_la_situacion_mundial_de_la_prevencion_ de_la_violencia.pdf. Consultado el 9 de marzo de 2018.
} 
En América Latina, siete países habían aprobado, hasta 2012, leyes que tipifican este delito: Chile, Costa Rica, Guatemala, México, Perú, El Salvador y Nicaragua. Al referirse a tales leyes, Nadine Gasman señaló que:

La tipificación de este delito obedece a la obligación de los Estados de adecuar sus legislaciones a los instrumentos internacionales pero también al incremento del número de muertes de mujeres y la crueldad con que la que se producen, a la ausencia de tipos penales especiales para describir adecuadamente el asesinato de mujeres basado en razones de odio, desprecio, y relaciones asimétricas de poder entre hombres y mujeres, así como a los altos índices de impunidad. ${ }^{27}$

La manera en que ha sido regulado en estas legislaciones difiere en cuanto a su denominación: Chile, Costa Rica, Guatemala y Nicaragua lo denominan femicidio, y El Salvador, México y Perú lo llaman feminicidio.

La diferencia entre uno y otro concepto se ha sustentado fundamentalmente en la impunidad. Al respecto, Marcela Lagarde ha desarrollado el concepto de feminicidio expresando que:

para diferenciarlo, preferí la voz feminicidio y denominar así al conjunto de violaciones a los derechos humanos de las mujeres que contienen los crímenes y las desapariciones de mujeres y que, estos fuesen identificados como crímenes de lesa humanidad El feminicidio es el genocidio contra mujeres y sucede cuando las condiciones históricas generan prácticas sociales que permiten atentados violentos contra la integridad, la salud, las libertades y la vida de niñas y mujeres. ${ }^{28}$

Y añade la autora:

Para que se dé el feminicidio concurren, de manera criminal, el silencio, la omisión, la negligencia y la colusión parcial o total de autoridades encargadas de prevenir y erradicar estos crímenes. Su ceguera de género

\footnotetext{
${ }^{27}$ Gasman, Nadine, "Presentación", La regulación del delito de Femicidio/Feminicidio en América Latina y El Caribe, Secretariado de la Campaña del Secretario General de las Naciones Unidas ÚNETE para poner fin a la violencia contra las mujeres, Ciudad de Panamá, Panamá, p. 7.

${ }^{28}$ Lagarde y de los Ríos, Marcela, "Antropología, feminismo y política: violencia feminicida y derechos humanos de las mujeres", Retos teóricos y nuevas prácticas, Margaret Bullen, Carmen Diez Mintegui (coordinadoras), pp. 216.
} 
o sus prejuicios sexistas y misóginos sobre las mujeres. Hay condiciones para el feminicidio cuando el Estado (o algunas de sus instituciones) no da las suficientes garantías a las niñas y las mujeres y no crea condiciones de seguridad que garanticen sus vidas en la comunidad, en la casa, ni en los espacios de trabajo de tránsito o de esparcimiento. Más aún, cuando las autoridades no realizan con eficiencia sus funciones. Cuando el Estado es parte estructural del problema por su signo patriarcal y por su preservación de dicho orden, el feminicidio es un crimen de Estado. ${ }^{29}$

Por su parte, Ana Carcedo conceptualiza el femicidio señalando que:

la violación, el incesto, el abuso físico y emocional, el acoso sexual, el uso de las mujeres en la pornografía, la explotación sexual, la esterilización o la maternidad forzada, etc., son todas expresiones distintas de la opresión de las mujeres y no fenómenos inconexos. En el momento en que cualquiera de estas formas de violencia resulta en la muerte de la mujer, ésta se convierte en femicidio. El femicidio es, por lo tanto, la manifestación más extrema de este continuum de violencia. ${ }^{30}$

No obstante esta disquisición teórica, en las legislaciones latinoamericanas se utilizan los dos términos, femicidio y feminicidio, para referirse a la muerte ocasionada a mujeres por razón de su género.

Por ejemplo, utilizan el término feminicidio: México, que en su Código Penal Federal, ${ }^{31}$ en el capítulo V, se refiere al feminicidio (reformada la denominación mediante decreto publicado en el Diario Oficial de la Federación el 14 de junio de 2012); El Salvador, Ley Especial. Integral para una Vida Libre de Violencia para las Mujeres, DECRETO 520, publicado en el Diario Oficial núm. 2, tomo 390, del 4 de enero de 2011. ${ }^{32}$

Otras legislaciones utilizan el término femicidio, por ejemplo: el Código Penal de Chile, ${ }^{33}$ en su artículo 390; Costa Rica, en la Ley de Penalización

\footnotetext{
${ }^{29}$ Ibid., pp. 216-217.

${ }^{30}$ Carcedo, Ana, op. cit., p. 14.

${ }^{31}$ Instituto de Investigaciones Juridicas, UNAM. Disponible en: https://www.juridicas.unam.mx/legislacion/ordenamiento/codigo-penal-federal. Consultado el 27 de enero de 2017.

32 Procuraduría General de la República. Disponible en: http://www.pgr.gob.sv/genero/descargas/ley\%20especial\%20integra|\%20para\%20una\%20vida\%20libre\%20de\%20violencia\%20para\%20las\%20mujeres_web.pdf. Consultado el 27 de enero de 2017.

${ }^{33}$ Biblioteca del Congreso Nacional (CL). Disponible en: https://www.leychile.cl/Navegar?idNorma=1984غtidParte= 0\# parricidio0. Consultado el 27 de enero de 2017.
} 
de la Violencia Contra las Mujeres, Ley 8589, del 25 de abril de 2007, ${ }^{34}$ en el artículo 21; Guatemala, en la Ley contra el Femicidio y otras Formas de Violencia contra la Mujer, en el decreto número $22-2008 ;{ }^{35}$ Nicaragua, en la Ley 779, Integral contra la Violencia hacia las Mujeres y de Reformas a la Ley 641, del Código Penal ${ }^{36}$, en el artículo 9.

Importante es mencionar que Colombia reformó su Código Penal e incorporó, vía circunstancia de agravación, el homicidio cometido "contra una mujer por el hecho de ser mujer”. En el caso de Argentina, mediante la Ley 26.791, se incorporó al artículo 80 del Código Penal la imposición de reclusión perpetua o prisión perpetua al que mate a una mujer cuando el hecho sea perpetrado por un hombre y mediare violencia de género.

\section{La tiPificación del FEMicidio EN ECUAdor}

La muerte violenta de una mujer es la forma más extrema de violencia de género, y en Ecuador está tipificada como femicidio, por lo que es importante enfatizar que un hecho de esta naturaleza tiene un trasfondo de género, de poder y de dominación que determina que la víctima sea una mujer. Por lo tanto, no es un hecho violento aislado, sino que responde a una construcción social patriarcal. ${ }^{37}$

El Ecuador, en el marco de sus obligaciones internacionales en materia de derechos humanos, tipificó el femicidio en el Código Orgánico Integral Penal (coIP) vigente desde agosto de 2014. Este nuevo delito permite al Estado investigar, juzgar y sancionar la muerte de las mujeres en razón de su género. Contando con la norma penal, corresponde a la Fiscalía titular de la acción pública aplicar la norma y poner en movimiento la administración de justicia, como en efecto lo ha hecho: ${ }^{38}$

\footnotetext{
${ }^{34}$ Sistema Costarricense de Información Jurídica. Disponible en: http://www.pgrweb.go.cr/scij/Busqueda/Normativa/Normas/nrm_norma.aspx?param1=NRMEtnValor1=1\&tnValor2=60183\&nValor3=105224\&tstrTipM=FN. Consultado el 2 de febrero de 2017.

${ }_{35}$ Disponible en: http://www.wpanet.org/uploads/News-Zonal-Representatives/wpa-policy-papers-from-zone3/ Zone\%203-Ley_contra_el_Femicidio_y_otras_Formas_de_Violencia_Contra_la_Mujer_Guatemala.pdf.

${ }_{36}$ Poder Judicial, República de Nicaragua. Disponible en: http://www.poderjudicial.gob.ni/pjupload/leyes/Ley_ No_779_Ley_Integral_Contra_la_Violencia_hacia_la_Mujer.pdf. Consultado el 26 de diciembre de 2016.

${ }^{37}$ Consejo Nacional para la Igualdad de Género Disponible en: http://igualdadgenero.gob.ec/images/docs/Comunicac-2017/Pronunciamiento\%20Enero\%202017.jpg [Consulta: 1 de febrero de 2017]

${ }^{38}$ Fiscalia General del Estado, Femicidio. Análisis penológico 2014-2015, 1ra edición digital, 2016, Quito, Ecuador, p. 8.
} 
Código Orgánico Integral Penal

Capitulo Segundo

DELITOS CONTRA LOS DERECHOS DE LIBERTAD

Sección Primera

DELITOS CONTRA LA INVIOLABILIDAD DE LA VIDA

Art. 141.-Femicidio.- La persona que, como resultado de relaciones de poder manifestadas en cualquier tipo de violencia, dé muerte a una mujer por el hecho de serlo o por su condición de género, será sancionada con pena privativa de libertad de veintidós a veintiséis años.

Art. 142.- Circunstancias agravantes del femicidio.- Cuando concurran una o más de las siguientes circunstancias se impondrá el máximo de la pena prevista en el artículo anterior:

1. Haber pretendido establecer o restablecer una relación de pareja o de intimidad con la víctima.

2. Exista o haya existido entre el sujeto activo y la víctima relaciones familiares, conyugales, convivencia, intimidad, noviazgo, amistad, compañerismo, laborales, escolares o cualquier otra que implique confianza, subordinación o superioridad.

3. Si el delito se comete en presencia de hijas, hijos o cualquier otro familiar de la víctima.

4. El cuerpo de la víctima sea expuesto o arrojado en un lugar público.

Como se ha señalado por la Fiscalía General del Estado:

Un nuevo tipo penal independiente se justifica solamente si ese delito va a proteger un bien jurídico diferente que justifique su existencia separado de otras figuras penales similares y de carácter neutro. En la especie, el homicidio es un tipo penal neutro que protege el bien jurídico "vida", entonces se podría pensar que este tipo penal es suficiente para proteger la vida de las mujeres que mueren por razones de género. Si aceptamos este razonamiento estaríamos olvidándonos que solamente las mujeres soportan femicidio o feminicidio y que el Estado está obligado a garantizarles el derecho a la vida, a una vida sin violencia, a la integridad personal, a la igualdad y no discriminación. Un tipo penal independiente permite distinguir las muertes que se producen por razones de género de otras muertes violentas. El femicidio o feminicidio se incluye en el 
catálogo de delitos para que el Estado proteja el derecho a la vida de las mujeres. $^{39}$

La inclusión de este delito en la legislación penal ecuatoriana es indudablemente la respuesta a un problema que ha adquirido dimensiones en el país, y pese a la existencia real de delitos cuyas víctimas directas son las mujeres, la legislatura demoró en introducir la figura penal en el catálogo de delitos. En vista de que no existía una norma punitiva específica, no se contaba tampoco con mecanismos de investigación y sanción de los ataques, la sistematicidad y la reincidencia, convirtiendo así en ineficaz la protección integral de los bienes jurídicos tutelados: la vida y la integridad personal de las mujeres.

Las normas internacionales sobre derechos humanos, como la Convención de Eliminación de Todas las Formas de Discriminación contra la Mujer, la Convención Interamericana de Belem do Pará, la Declaración y Programa de Acción de Viena, las corrientes socio-culturales que incluyen el enfoque de género en las leyes y en la justicia, e igualmente las legislaciones de otros países de la región, junto a los hechos dolorosos que se han presentado en el país, debieron ser razones suficientes para la inclusión del femicidio en el ordenamiento jurídico interno penal ecuatoriano.

La Comisión Especializada de Justicia y Estructura del Estado de la Asamblea Nacional, en el informe para el segundo debate del proyecto del Código Orgánico Integral Penal (coIP), de fecha 4 de octubre del 2013, reconoce que la normativa internacional que se relaciona con el tema de la violencia o discriminación contra la mujer es el antecedente para tipificar el femicidio, y lo califica como un avance para luchar contra la violencia que sufren las mujeres. Por consiguiente, para el órgano legislativo este nuevo delito se encontraría intimamente ligado con la violencia en contra de las mujeres.

$\mathrm{Al}$ respecto, conviene previamente delimitar la corriente que adopta el CoIP que entró en vigencia en agosto del 2014, para analizar la norma que describe y sanciona el femicidio, y es así como, al tenor del artículo 18, el delito en general responde a una noción dogmática, por lo que contiene todas las características de la acción sancionada con una pena y, por tanto, es la conducta típica, antijurídica y culpable. En ese orden, el artículo 25, al tratar de la tipicidad, consigna que los tipos penales describen los elementos de las conductas penalmente relevantes; en consecuencia, es la descripción

39 Ibid., p. 11. 
concreta de la conducta prohibida integrada por una parte objetivada y otra subjetiva.

Del texto del artículo 141 del coIP, transcrito up supra, se infiere que los elementos objetivos del tipo son:

- $\quad$ El sujeto activo del delito: es la persona que lleva a cabo la conducta tipificada en esa norma, lo que significa que no es un sujeto calificado, pues no se exige que reúna ciertas calidades especiales, como sucede en las legislaciones de otros países.

- El sujeto pasivo del delito: es el titular del bien jurídico protegido, que en ocasiones puede tener ciertas calidades, como en el femicidio. El artículo 141 establece que es una mujer, por consiguiente la conducta o acción debe dirigirse en su contra y será sólo ella quien reciba el perjuicio por parte de cualquier persona.

- La acción o conducta: es el núcleo del tipo y se identifica como verbo rector; en el caso es matar. Está seguido por el resultado, que es el efecto y la consecuencia manifestada en el mundo exterior, y que incide tanto en el plano físico como en el psíquico.

- $\quad$ El bien jurídico: El bien jurídico permite descubrir la naturaleza del tipo, en tal forma que le da sentido y fundamento; es el bien tutelado por el derecho penal. En el femicidio: la vida de la mujer.

- Los elementos normativos: implican una valoración, y tienen por ello cierto grado de subjetivismo. En algunos casos se relacionan con la ley o instrumentos internacionales, mientras que en otros hay contenido cultural y están determinados con la sociología o la teoría política.

El artículo 141 tiene como elementos normativos las relaciones de poder manifestadas en cualquier tipo de violencia, la condición de mujer, la condición de género. Al efecto, la Convención Interamericana para Prevenir, Sancionar y Erradicar la Violencia en contra de la Mujer delimita los tipos de violencia, y es claro que es resultado de un abusivo ejercicio del poder y de inequidad en las relaciones hombre-mujer.

Pero como ya se ha señalado, la condición de género como circunstancia motivante del femicidio es una construcción cultural y social que va más allá de las diferencias sexuales. La norma legal debe ser interpretada teniendo en cuenta el método histórico de interpretación; en ese sentido, no solamente se 
ha de revisar detenidamente el texto del artículo, sino que además se investigarán los criterios que el legislador imprimió en la redacción de la norma, en los debates previos y los antecedentes. Por esa razón, haber utilizado la conjunción $o$ para separar las causas determinantes para dar muerte a una mujer y configurar de esa manera el femicidio podría considerarse un error, porque está creándose un delito abierto.

En realidad, la condición de mujer no es equiparable a la condición de género, porque es más amplia y su aplicación requiere de interpretaciones y razonamientos que en cada caso deberán hacer el fiscal y el juzgador, para tener claridad en la visión de género, que desde luego no tiene tan sólo el componente de la diferencia sexual.

En cualquiera de las formas de interpretación de la norma, aparece que el sujeto activo del delito puede ser cualquier persona, y que el sujeto pasivo es siempre una mujer, por lo que el ataque al bien jurídico vida puede provenir de un hombre, de otra mujer o de alguien que tenga diferente preferencia sexual. En consecuencia, es preciso analizar las relaciones de poder aun entre mujeres, como de la madre sobre la hija o de la empleadora frente a sus empleadas, por ejemplo.

El artículo 141 no asume todas las categorías del femicidio: íntimo, no intimo y por conexión. Además, las diversas circunstancias en las cuales se puede cometer el delito están ubicadas en el artículo 142 en calidad de dos agravantes, y no como constitutivas del delito, lo que daría lugar a que algunas conductas quedarían por fuera del tipo.

\section{Conclusiones}

Sólo la transformación de los cánones establecidos en nuestras sociedades en cuanto a los roles de hombres y mujeres y una verdadera conciencia sobre la igualdad pueden contribuir a erradicar esta forma de violencia extrema.

La regulación del femicidio en el Código Orgánico Integral Penal ecuatoriano, así como en otras legislaciones de América Latina, es una muestra del compromiso del Estado con la eliminación de la violencia contra la mujer, pero no ha tenido el impacto necesario, teniendo en cuenta que los crímenes de este tipo siguen aumentando. Las limitaciones que presenta la actual regulación de este tipo penal en Ecuador permiten afirmar que el perfeccionamiento de la regulación del delito de femicidio en el coIP puede contribuir a enfrentar de modo más adecuado este flagelo que está presente en la sociedad. 


\section{Referencias}

Arboleda, María, Cuaderno de Trabajo Plan de Igualdad, No Discriminación y Buen Vivir para las Mujeres Ecuatorianas. Marco conceptual, ruta metodológica y estrategia de transversalización 2010-2014, primera edición, Comisión de Transición hacia el Consejo de las Mujeres y la Igualdad de Género, Quito, 2011.

Arroyo, Roxana, Violencia Estructural de Género una Categoría Necesaria de Análisis para los Derechos Humanos de las Mujeres. Disponible en: http:// observatoriojyg.org/index.php/280-doctrina/1-derecho-de-humanas/2igualdad/686-violencia-estructural-de-genero-una-categoria-necesaria-de-analisis-para-los-derechos-humanos-de-las-mujeres. Consultado el 26 de enero de 2017.

AR\#NiUnaMujerMenosCR. Disponible en: http://www.ameliarueda.com/especiales/ feminicidios/. Consultado el 2 de febrero de 2017.

Asamblea Nacional de la República del Ecuador, Ley Orgánica Integral para Prevenir y Erradicar la Violencia contra las Mujeres, Registro Oficial 175, Quito, 5 de febrero de 2018.

Biblioteca del Congreso Nacional (CL). Disponible en: https://www.leychile.cl/Nav egar?idNorma=1984CtidParte=0\#parricidio0. Consultado el 27 de enero de 2017.

Carcedo, Ana, Femicidio en Costa Rica 1990-1999, INAMU, San José, Costa Rica, 2000, p. 13.

Consejo Nacional de Igualdad de Género, Agenda Nacional de las Mujeres y la Igualdad de Género 2014-2017, El Telégrafo, Quito, 2014.

Consejo Nacional para la Igualdad de Género. Disponible en: http://igualdadgenero. gob.ec/images/docs/Comunicac-2017/Pronunciamiento\%20Enero\%20 2017.jpg. Consultado el 1 de febrero de 2017.

Convención Interamericana para Prevenir, Sancionar y Erradicar la Violencia contra la Mujer Convención de Belém do Pará. Disponible en: https://www. unicef.org/argentina/spanish/ar_insumos_ConvencionBelem.pdf. Consultado el 27 de enero de 2017.

Economíahoy.mex. Disponible en: http://www.economiahoy.mx/nacionaleAmmx/ noticias/7406635/03/16/Siete-mujeres-mueren-al-dia-en-Mexico-victimas-de-la-violencia.html. Consultado el 20 de diciembre de 2016.

El Universo. Disponible en: http://www.eluniverso.com/noticias/2017/01/24/ nota/6014020/casos-femicidos-ecuador-se-han-duplicado-2017-segundinased. Consultado el 1 de febrero de 2017.

Encuesta Nacional de Relaciones Familiares y Violencia de Género. Disponible en: http://www.igualdadgenero.gob.ec/estad\%C3\%ADsticas/57-encuestanacional-de-relaciones familiares-y-violencia-de-g\%C3\%A9nero.html. Consultado el 26 de diciembre de 2016. 
Femicidio.net. Disponible en: http://www.feminicidio.net/articulo/ni-menos-lasociedad-argentina-indignada-la-ola femicidios-azota-al-pa\%C3\%ADs. Consultado el 20 de diciembre de 2016.

Fiscalía General del Estado, Femicidio. Análisis penológico 2014-2015, 1ra edición digital, 2016, Quito, Ecuador, p. 8.

Fiscalía General del Estado. Disponible en: fiscalia.gob.ec/informativo/Discurso FGE_Rendicion_Cuentas_2016.pdf. Consultado el 26 de enero de 2017.

Fríes, Lorena; Hürtado, Victoria, Estüdio de la información sobre la violencia contra la mujer en América Latina y el Caribe, CEPAL, División de Asuntos de Género, Santiago de Chile, 2010. Disponible en: https://repositorio.cepal. org/bitstream/handle/11362/5826/1/S0900880_es.pdf. Consultado el 9 de marzo de 2018.

Garita Vílchez, Ana Isabel, La regulación del delito de Femicidio/Feminicidio en América Latina y El Caribe, Secretariado de la Campaña del Secretario General de las Naciones Unidas ÚNETE para poner fin a la violencia contra las mujeres, Ciudad de Panamá, p. 15.

Herdoíza, Magdalena, Construyendo igualdad en la educación superior. fundamentación y lineamientos para transversalizar los ejes de igualdad y ambiente, 1ra edición, Senescyt/Unesco, Quito, 2015, p. 46.

INEC. Disponible en: http://www.ecuadorencifras.gob.ec//documentos/web-inec/ Uso Tiempo/Presentacion \%20Principales Resultados.pdf. Consultado el 20 de diciembre de 2016.

Instituto de Investigaciones Jurídicas, UNAM. Disponible en: https://www.juridicas. unam.mx/legislacion/ordenamiento/codigo-penal-federal. Consultado el 27 de enero de 2017.

Ley contra el Femicidio y otras Formas de Violencia contra la Mujer. Disponible en: http://www.wpanet.org/uploads/News-Zonal-Representatives/wpapolicy-papers-from-/Zone\%203Ley_contra_el_Femicidio_y_otras_Formas_de_Violencia_Contra_la_Mujer_Guatemala.pdf.

Ministerio Coordinador de Desarrollo Social. Disponible en: http://www.desarrollosocial.gob.ec/wp-content/uploads/downloads/2015/03/RENDICIONCUENTAS-20141.pdf, pp. 15-16. Consultado el 26 de enero de 2016.

ONU, Informe sobre la situación mundial de la prevención de la violencia 2014 , Washington, D. C., 2016, p. 40. Disponible en: https://oig.cepal.org/sites/ default/files/informe_sobre_la_situacion_mundial_de_la_prevencion_ de la violencia.pdf. Consultado el 9 de marzo de 2018.

Poder Judicial. República de Nicaragua. Disponible en: http://www.poderjudicial. gob.ni/pjupload/leyes/Ley_No_779_Ley_Integral_Contra_la_Violencia_ hacia la Mujer.pdf. Consultado el $2 \overline{6}$ de diciembre de $201 \overline{6}$.

Procuraduría General de la República. Disponible en: http://www.pgr.gob.sv/genero/descargas/ley\%20especial\%20integral\%20para\%20una\%20vida\%20 libre\%20de\%20violencia\%20para\%20las\%20mujeres_web.pdf. Consultado el 27 de enero de 2017. 
Sistema Costarricense de Información Jurídica. Disponible en: http://www.pgrweb. go.cr/scij/Busqueda/Normativa/Normas/nrm_norma.aspx?param 1=NR MEtnValor1=1\&tnValor2=60183\&tnValor3=105224\&tstrTipM=FN. Consultado el 2 de febrero de 2017. 
